\title{
Trichomonas vaginalis Induces SiHa Cell Apoptosis by NF- $\kappa$ B Inactivation via Reactive Oxygen Species
}

\author{
Juan-Hua Quan,, Byung-Hun Kang, ${ }^{2}$ Jung-Bo Yang, ${ }^{2}$ Yun-Ee Rhee, ${ }^{2}$ Heung-Tae Noh, \\ In-Wook Choi, ${ }^{3}$ Guang-Ho Cha, ${ }^{3}$ Jae-Min Yuk, ${ }^{3}$ and Young-Ha Lee ${ }^{3}$ \\ ${ }^{1}$ Department of Gastroenterology, Affiliated Hospital of Guangdong Medical University, Zhanjiang, \\ Guangdong Province 524001, China \\ ${ }^{2}$ Department of Obstetrics and Gynecology, Chungnam National University School of Medicine, Daejeon 35015, Republic of Korea \\ ${ }^{3}$ Department of Infection Biology, Chungnam National University School of Medicine, Daejeon 35015, Republic of Korea \\ Correspondence should be addressed to Young-Ha Lee; yhalee@cnu.ac.kr
}

Received 18 August 2017; Revised 7 November 2017; Accepted 19 November 2017; Published 18 December 2017

Academic Editor: Marlene Benchimol

Copyright (c) 2017 Juan-Hua Quan et al. This is an open access article distributed under the Creative Commons Attribution License, which permits unrestricted use, distribution, and reproduction in any medium, provided the original work is properly cited.

\begin{abstract}
Trichomonas vaginalis induces apoptosis in host cells through various mechanisms; however, little is known about the relationship between apoptosis, reactive oxygen species (ROS), and NF- $\kappa$ B signaling pathways in the cervical mucosal epithelium. Here, we evaluated apoptotic events, ROS production, and NF- $\kappa \mathrm{B}$ activity in T. vaginalis-treated cervical mucosal epithelial SiHa cells, with or without specific inhibitors, using fluorescence microscopy, DNA fragmentation assays, subcellular fractionation, western blotting, and luciferase reporter assay. SiHa cells treated with live T. vaginalis at a multiplicity of infection of 5 (MOI 5 ) for $4 \mathrm{~h}$ produced intracellular and mitochondrial ROS in a parasite-load-dependent manner. Incubation with T. vaginalis caused DNA fragmentation, cleavage of caspase 3 and PARP, and release of cytochrome $c$ into the cytoplasm. T. vaginalis-treated SiHa cells showed transient early NF- $\kappa$ B p 65 nuclear translocation, which dramatically dropped at $4 \mathrm{~h}$ after treatment. Suppression of NF- $\kappa \mathrm{B}$ activity was dependent on parasite burden. However, treatment with the ROS scavenger, $\mathrm{N}$-acetyl-C-cysteine (NAC), reversed the effect of T. vaginalis on apoptosis and NF- $\kappa \mathrm{B}$ inactivation in SiHa cells. Taken together, T. vaginalis induces apoptosis in human cervical mucosal epithelial cells by parasite-dose-dependent ROS production through an NF- $\kappa$ B-regulated, mitochondria-mediated pathway.
\end{abstract}

\section{Introduction}

Trichomonas vaginalis, a flagellated lumen-dwelling protozoan parasite, causes a sexually transmitted disease, with an estimated 275 million new cases worldwide each year [1]. This parasite causes vaginitis and cervicitis in women and asymptomatic urethritis and prostatitis in men, with associated morbidities including endometritis, preterm birth, and viral infections [2,3]. Epidemiological studies have also shown that $T$. vaginalis acts as a cofactor in transmitting human immunodeficiency virus (HIV) type 1 , and infection of women with $T$. vaginalis increases the risk of HIV infection [4]. However, the pathophysiology of trichomoniasis is not well established.

Apoptosis, a biological event induced by the activation of a series of enzymes known as caspases, may occur when a cell is damaged beyond repair, infected with a pathogen, or stressed due to DNA damage or toxic chemicals. Studies using in vitro models have shown that $T$. vaginalis induces apoptosis of various types of cells, including a murine monocyte/macrophage cell line [5-7], human monocyte-derived macrophages $[8,9]$, primary human vaginal epithelial cells $[10,11]$, human cervical cancer cells ( $\mathrm{SiHa}$ cells), and vaginal epithelial cells (MS74 cells) [12]. Although there are some reports of apoptosis induced by T. vaginalis, the mechanisms of apoptosis in human cervical mucosal epithelial cells infected with $T$. vaginalis are not well elucidated.

NF- $\kappa \mathrm{B}$ is an essential transcription factor that regulates diverse physiological processes, including cell growth, differentiation, inflammatory responses, and apoptosis [13]. In normal conditions, NF- $\kappa$ B remains in an inactive form in the 
cytoplasm in association with the inhibitory family of $\mathrm{I} \kappa \mathrm{B}$ molecules. Upon stimulation, $\mathrm{I} \kappa \mathrm{B}-\alpha$ is phosphorylated by $\mathrm{I} \kappa \mathrm{B}$ kinase (IKK) and degraded, leading to nuclear translocation of NF- $\kappa \mathrm{B}$ p 65 and p50 to induce the expression of various genes controlling inflammatory and immune responses [14]. Plumbagin has been shown to inhibit cell growth and potentiate apoptosis in human GC cells through the NF- $\kappa \mathrm{B}$ pathway [15]. T. vaginalis inhibits proinflammatory cytokine production in macrophages by suppressing NF- $\kappa \mathrm{B}$ activation [16]. However, there are no data on the role of NF- $\kappa \mathrm{B}$ signaling pathways in cervical epithelial $\mathrm{SiHa}$ cells in response to $T$. vaginalis.

The cervicovaginal mucosa is the first line of defense against pathogenic organisms. Reactive oxygen species (ROS) are essential for many biological functions including apoptosis and NF- $\kappa \mathrm{B}$ activation and are known for their ability to regulate certain essential signaling pathways. Their targets include protein kinase B (Akt), nuclear factor kappa-lightchain enhancer of activated B cells (NF- $\kappa \mathrm{B})$, and mitogenactivated protein kinases (MAPKs) [17]. ROS also function as intermediates in cellular processes such as inflammatory responses, cell cycle progression, apoptosis, aging, and cancer $[17,18]$. Previous reports have shown that dioscin (a natural product) and casticin (one of the main components of Fructus viticis) induce apoptosis of $\mathrm{HeLa}$ and $\mathrm{SiHa}$ cells by promoting ROS-mediated DNA damage and the mitochondrial signaling pathway $[19,20]$. Activation of MAPK is required for ROS generation and exocytosis in HMC-1 cells by T. vaginalisderived secretory products [21]. T. vaginalis induces IL-1 $\beta$ production in human prostate epithelium through activation of ROS [22]. There are numerous reports of ROS production or ROS function in various cell lines or in response to stimulants. However, the effect of ROS in T. vaginalis-treated cervical mucosal epithelial cells has not been described to date. Specifically, the roles of ROS in relation to the NK- $\kappa \mathrm{B}$ signaling pathway for apoptosis induction in T. vaginalistreated cervical mucosal epithelial cells are unclear. Thus, to elucidate this mechanism, human cervical mucosal epithelial SiHa cells were treated with $T$. vaginalis, and apoptotic features, ROS production, and NF- $\kappa \mathrm{B}$ activity were evaluated using fluorescence microscopy, DNA fragmentation assays, subcellular fractionation, western blotting, and luciferase assays.

\section{Materials and Methods}

2.1. Antibodies and Reagents. Antibodies to poly-(ADPribose) polymerase (PARP), caspase 3, cleaved caspase 3, cytochrome $c, \mathrm{NF}-\kappa \mathrm{B}$ p65, Bcl-2, COX IV, Histone H3, and $\beta$-actin were purchased from Cell Signaling Technology (Danvers, MA, USA). Mouse monoclonal anti- $\alpha$-tubulin was purchased from Santa Cruz Biotechnology (Santa Cruz, CA, USA). N-Acetyl-L-cysteine (NAC), dihydroethidium (DHE), MitoSOX $^{\text {тм }}$ Red, 3-(4,5-dimethyl-2-thiazolyl)-2,5-diphenyl$2 \mathrm{H}$-tetrazolium bromide (MTT), and staurosporine (STS) were purchased from Sigma-Aldrich Chemical Co. (St. Louis, MO, USA).
2.2. Live T. vaginalis Culture. The T016 strain of $T$. vaginalis was cultured in a glass, screw-capped tube containing Diamond's trypticase-yeast extract-maltose (TYM) medium (NAPCO, Winchester, VA, USA) supplemented with $10 \%$ heat-inactivated horse serum (Sigma-Aldrich) in $5 \% \mathrm{CO}_{2}$ at $37^{\circ} \mathrm{C}$ for $24 \mathrm{~h}$ [12]. Cultured parasites were monitored for motility and the viability of $T$. vaginalis was determined before each experiment using trypan blue staining (>99\%).

\subsection{Preparation of $T$. vaginalis Excretory/Secretory Product} (ESP). T. vaginalis ESP were prepared as described previously [12]. To prepare the T. vaginalis ESP, freshly purified trophozoites $\left(1 \times 10^{7}\right.$ cells $\left./ \mathrm{mL}\right)$ were incubated with TYM medium at $37^{\circ} \mathrm{C}$ for $1 \mathrm{~h}$ in $5 \% \mathrm{CO}_{2}$. After centrifugation for $30 \mathrm{~min}$ at $10,000 \mathrm{~g}$, the ESP-containing supernatant was filtered through a $0.2 \mu \mathrm{m}$ pore filter and stored at $-70^{\circ} \mathrm{C}$. The T. vaginalis ESP concentrations were determined by the Bradford assay with bovine serum albumin (BSA) as the standard.

2.4. Culture of SiHa Cells. The human cervical mucosal epithelial cancer cell line, SiHa, was obtained from the American Type Culture Collection (ATCC, Manassas, VA, USA) and maintained in Dulbecco's Modified Eagle's Medium (DMEM) supplemented with 10\% heat-inactivated fetal bovine serum (FBS; Gibco BRL, Grand Island, NY, USA) and antibiotic-antimycotic solution (Gibco BRL) in a $5 \% \mathrm{CO}_{2}$ atmosphere at $37^{\circ} \mathrm{C}$.

2.5. Study Design. SiHa cells were seeded on 96-well plates (for MTT assay), 12-well cover-slips (for ROS detection), or $100 \mathrm{~mm}$ culture dishes (for western blotting, DNA fragmentation assay, and transfection) at various concentrations and grown to confluence at $37^{\circ} \mathrm{C}$ in $5 \% \mathrm{CO}_{2}$.

We first evaluated ROS production in T. vaginalistreated SiHa cells by fluorescence microscopy using DHE and MitoSox. Next, we investigated the induction of apoptosis in SiHa cells after T. vaginalis treatment by DNA fragmentation assay and western blotting. Also, NF- $\kappa \mathrm{B}$ activity was determined in $T$. vaginalis-treated cells by western blotting and luciferase reporter assay. To investigate the role of ROS in inducing apoptosis, $T$. vaginalis-treated $\mathrm{SiHa}$ cells were pretreated with a selective ROS scavenger, NAC, for $30 \mathrm{~min}$, and then evaluated for apoptotic features and NF- $\kappa$ B activity. The control group was just $\mathrm{SiHa}$ cell not treated with $T$. vaginalis or T. vaginalis ESP. Each experiment was performed at least three times.

2.6. Cell Viability Assay. Cell viability was determined using an MTT assay. Briefly, cells were seeded at a density of $5 \times$ $10^{3}$ cells/well in 96-well plates. The plates were incubated at $37^{\circ} \mathrm{C}$ for $24 \mathrm{~h}$ to adhere the SiHa cells to the bottom of the well. Different concentrations of NAC $(0.1,1,10 \mathrm{mM})$ were then added to each well of 96-well plates for $24 \mathrm{~h}$. Then, $10 \mu \mathrm{L}$ of MTT solution $(5 \mathrm{mg} / \mathrm{mL})$ was added and cells were further incubated at $37^{\circ} \mathrm{C}$ for $4 \mathrm{~h}$. Following the removal of MTT solution, water-insoluble formazan was dissolved by adding $100 \mu \mathrm{L}$ of dimethyl sulfoxide (DMSO) to each well. Finally, optical density (OD) was measured at $570 \mathrm{~nm}$ using 
a microplate reader (Sunnyvale, CA, USA). Data were shown as the absorbance relative to the untreated control.

2.7. DNA Fragmentation. DNA was isolated from $1 \times 10^{6}$ SiHa cells using a genomic DNA extraction kit (iNtRON Biotechnology, Seoul, Korea). An equal amount of DNA was loaded into each well of $2 \%$ agarose gels containing ethidium bromide $(0.5 \mu \mathrm{g} / \mathrm{mL})$ and separated electrophoretically using Tris-borate-EDTA ( $\mathrm{pH}$ 8.0) as the running buffer ( $89 \mathrm{mM}$ Tris-borate, $2 \mathrm{mM}$ EDTA). Migrating DNA bands were visualized with a UV transilluminator (Gel Doc, BioRad Laboratories, Ltd., Hercules, CA, USA).

\subsection{Measurement of ROS Generation}

(1) DHE. Intracellular ROS generation was detected by fluorescence microscopy using DHE. SiHa cells $\left(1 \times 10^{5}\right.$ cells/well; 12 -well plate) seeded on a cover-slip were cultured in DMEM supplemented with $10 \%$ FBS and the culture medium was replaced when cells reached $80 \%$ confluence. To evaluate the generation of ROS, SiHa cells were treated with live $T$. vaginalis (MOI 1 and 5) and T. vaginalis excretory/secretory product (ESP, 20 and $100 \mu \mathrm{g} / \mathrm{mL}$ ) for $0.5 \mathrm{~h}, 1 \mathrm{~h}$, and $4 \mathrm{~h}$. After incubation, culture supernatants were removed and cells were washed twice with $1 \times$ PBS before incubating with $10 \mu \mathrm{M}$ DHE in fresh medium, free of FBS, at $37^{\circ} \mathrm{C}$ for $30 \mathrm{~min}$ in the dark. After washing with HBSS buffer, intracellular ROS, as indicated by DHE fluorescence, was detected with a fluorescence microscope (Olympus BX51, Japan).

(2) Mitochondrial Superoxide Detection. Mitochondrial superoxide formation was detected by fluorescence microscopy using MitoSOX Red as a specific fluorescent probe. SiHa cells $\left(1 \times 10^{5}\right.$ cells/well; 12 -well plate $)$ seeded on a coverslip were cultured in DMEM supplemented with 10\% FBS and the culture medium was replaced when the cells reached $80 \%$ confluence. To evaluate the generation of ROS, SiHa cells were treated with live T. vaginalis (MOI 1 and 5) and T. vaginalis ESP $(20$ and $100 \mu \mathrm{g} / \mathrm{mL}$ ) for $0.5 \mathrm{~h}, 1 \mathrm{~h}$, and $4 \mathrm{~h}$. After incubation, culture supernatants were removed and cells were washed twice with $1 \times$ PBS. MitoSOX staining was performed according to the manufacturer's protocol. Briefly, cells were incubated with $1 \mathrm{~mL}$ of $5 \mu \mathrm{M}$ of the MitoSOX reagent probe in the dark (covered with foil) and placed at $37^{\circ} \mathrm{C}$ in a humidified incubator with $5 \% \mathrm{CO}_{2}$ for $10 \mathrm{~min}$. After incubation, cells were washed thoroughly with warm PBS and mounted for imaging. MitoSOX Red was visualized using a fluorescence microscope (Olympus BX51). All experiments were performed on triplicate samples and fluorescence intensity was calculated using Image J software, and graph was plotted using SigmaPlot 12.5 (Systat Software, San Jose, CA).

2.9. Subcellular Fractionation. Isolation of cytosol, mitochondrial, and nuclear subcellular fractions were performed as described previously with minor modifications [23]. Cytosolic extracts free of nuclei and mitochondria were prepared as follows. Briefly, cells were washed in ice-cold PBS ( $\mathrm{pH} 7.2$ ) and then in hypotonic extraction buffer (HEB; $50 \mathrm{mM}$ PIPES, $50 \mathrm{mM} \mathrm{KCl}, 5 \mathrm{mM}$ EGTA, $2 \mathrm{mM} \mathrm{MgCl}_{2}$,
$1 \mathrm{mM}$ dithiothreitol, 0.1 mM PMSF, $\mathrm{pH}$ 7.4) and harvested by centrifugation. Pellets were resuspended in HEB and lysed in a Dounce homogenizer. These cell lysates were then centrifuged at $100,000 \mathrm{~g}$ for $60 \mathrm{~min}$ at $4^{\circ} \mathrm{C}$ and the supernatants were flash-frozen in cold ethanol and stored in aliquots at $-80^{\circ} \mathrm{C}$.

Nuclear and mitochondrial fractions were prepared by washing cells in ice-cold PBS and then resuspending in an isotonic homogenization buffer $(10 \mathrm{mM}$ Tris- $\mathrm{HCl}, \mathrm{pH}$ 7.5, 0.25 $\mathrm{M}$ sucrose, $10 \mathrm{mM} \mathrm{KCl,} 1 \mathrm{mM}$ EDTA, $1 \mathrm{mM}$ dithiothreitol, $0.1 \mathrm{mM}$ phenylmethylsulphonyl fluoride, EDTA-free complete cocktail of protease inhibitors [Roche, Switzerland]). After 60 strokes in a Dounce homogenizer, the unbroken cells were removed by centrifugation at $30 \mathrm{~g}$ for $10 \mathrm{~min}$. The nuclei were pelleted by centrifugation at $1,000 \mathrm{~g}$ for $10 \mathrm{~min}$ and supernatants were centrifuged at $14,000 \mathrm{~g}$ for $20 \mathrm{~min}$. The supernatants were collected as the mitochondrial fraction. The quality of the fraction experiments was confirmed by assessing the presence of $\alpha$-Tubulin, COX IV, and Histone $\mathrm{H} 3$ for cytosol, mitochondrial, and nuclear fraction, respectively.

2.10. Western Blotting. Equal amounts of fractioned proteins were separated by SDS-PAGE and transferred to a polyvinylidene difluoride membrane. The membranes were blocked in Tris-buffered saline (20 mM Tris, 137 mM NaCl, pH 7.6) containing 0.1\% Tween-20 (TBST) and 5\% skim milk. After being washed once in TBST, membranes were incubated overnight at $4^{\circ} \mathrm{C}$ with the primary antibodies diluted in TBST supplemented with 5\% BSA. The antibodies used were as follows: anti-caspase 3, anti-cleaved caspase 3, anti-PARP, anti-NF$\kappa \mathrm{B}$ p65, anti-Bcl-2, and anti-cytochrome $c$. Following three consecutive washes in TBST, membranes were incubated for $2 \mathrm{~h}$ with horseradish peroxidase-conjugated anti-mouse or anti-rabbit IgG (Santa Cruz Biotechnology) diluted 1:10,000 with incubation buffer, as described above. After extensive washing, bound secondary antibodies were visualized using an enhanced ECL chemiluminescence detection kit (GE Healthcare, Little Chalfont, UK).

2.11. Transfection and Luciferase Reporter Assay. The pGL3$\mathrm{NF}-\kappa \mathrm{B}$ promoter constructs (kindly provided by Dr. KyungCheol Sohn, Department of Dermatology, Chungnam National University) were transfected into SiHa cells with LipofectAmine 2000 (Invitrogen, Carlsbad, California, USA). Luciferase activity was assayed with a luciferase assay kit (Promega, Madison, WI, USA) described as follows. SiHa cells $\left(1 \times 10^{6}\right)$ were plated on $60 \mathrm{~mm}$ Petri dishes. After overnight incubation, cells were transfected with an NF- $\kappa \mathrm{B}$ reporter plasmid linked to a luciferase gene and kept for $48 \mathrm{~h}$, after which the medium was changed. Cells were treated with $T$. vaginalis for $2 \mathrm{~h}$. Cell extracts were then prepared in $500 \mu \mathrm{L}$ of $1 \times$ reporter lysis buffer. Lysates were centrifuged at $13,000 \mathrm{~g}$ for $5 \mathrm{~min}$ and luciferase activity was measured using a Microlumat LB96P Luminometer (Perkin Elmer, Wallac, Inc., USA). Luciferase activity was normalized relative to $\beta$-galactosidase activity in each sample. 

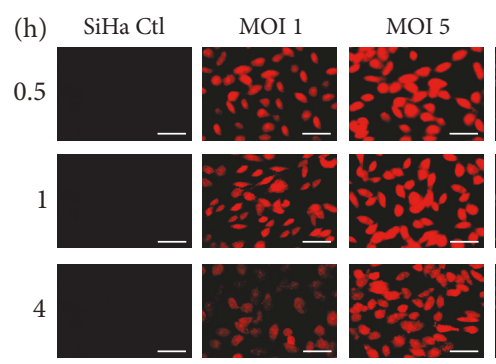

(a)
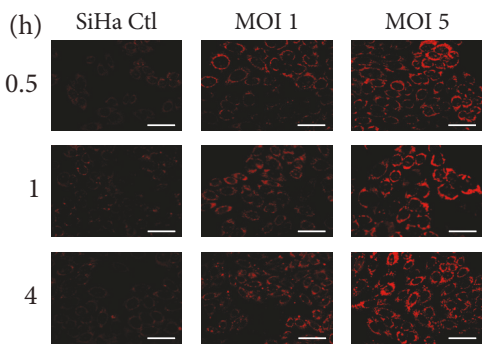

(c)

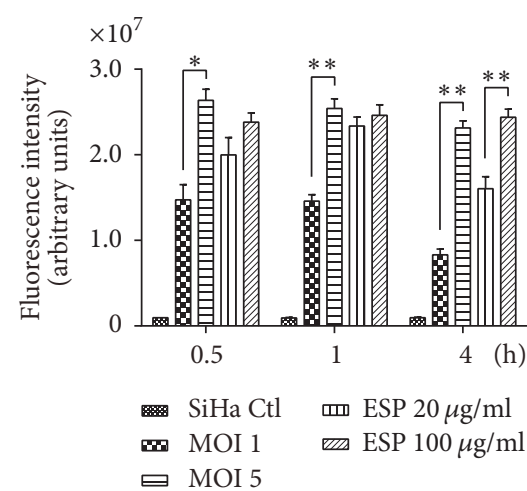

(b)

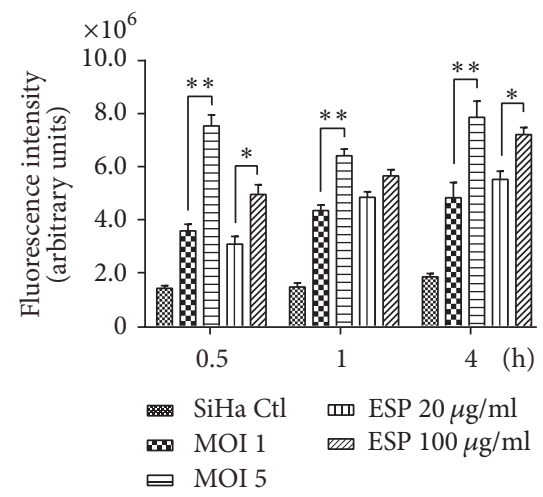

(d)

FIGURE 1: Trichomonas vaginalis T016 strain induced reactive oxygen species (ROS) production in human cervical mucosal epithelial SiHa cells, in parasite-burden-dependent manner. SiHa cells were treated with live T. vaginalis (multiplicity of infections 1 and 5) and T. vaginalis excretory/secretory products (ESP, 20 and $100 \mu \mathrm{g} / \mathrm{mL}$ ) at indicated time points. (a and b) Intracellular ROS production was detected by measurement of dihydroethidium (DHE) fluorescence. Intracellular ROS levels were significantly increased in a T. vaginalis parasite-burdendependent manner. ( $c$ and d) Mitochondrial ROS production was detected by MitoSOX reagent. Mitochondrial ROS production was significantly increased in parasite-burden- and ESP concentration-dependent manner. The experiment was repeated three times with similar results. Scale bars: $100 \mu \mathrm{m} ;{ }^{*} P<0.05,{ }^{* *} P<0.01$.

2.12. Statistical Analysis. Data were presented as means \pm SD. Statistical significance was determined by ANOVA (StatView; Abacus Concepts Inc., Berkeley, CA, USA). All experiments were performed at least in triplicate on separate days. Differences were considered significant at $P$ values $<0.05$.

\section{Results}

3.1. T. vaginalis Led to the Generation of Intracellular and Mitochondrial ROS in SiHa Cells, in Parasite-Burden-Dependent Manner. Oxidative stress is an important factor for toxicity associated with apoptosis. T. vaginalis resides in the vagina and colonizes the cervix. It attaches to human host epithelial cells to establish and maintain adhesion. To investigate whether ROS is generated in cervical mucosal epithelial SiHa cells after T. vaginalis treatment, intracellular and mitochondrial ROS levels were measured in SiHa cells following treatment with live T. vaginalis and T. vaginalis ESP.

Intracellular ROS levels were prominently increased in SiHa cells treated with live $T$. vaginalis and $T$. vaginalis ESP from $0.5 \mathrm{~h}$ after treatment. Intracellular ROS levels were significantly higher in cells treated with $T$. vaginalis
MOI 5 than those $T$. vaginalis MOI 1 from $0.5 \mathrm{~h}$ until $4 \mathrm{~h}$ posttreatment. In cells treated with $T$. vaginalis ESP, intracellular ROS levels were increased in ESP-concentrationdependent manner at $4 \mathrm{~h}$ after treatment, but not at 05 and $1 \mathrm{~h}$ (Figures 1(a) and 1(b)). Similarly, mitochondrial ROS were also produced after $0.5 \mathrm{~h}$ of incubation with live T. vaginalis and T. vaginalis ESP. Mitochondrial ROS production was increased in direct proportion to parasite burden and ESP concentration (Figures 1(c) and 1(d)). These findings suggest that $T$. vaginalis is a strong inducer of intracellular and mitochondrial ROS, in parasite-burden-dependent manner, and that $T$. vaginalis ESP has a similar effect to live $T$. vaginalis.

3.2. T. vaginalis Induced Apoptosis through an Intrinsic Mitochondrial Apoptotic Pathway in SiHa Cells. We aimed to identify the pathway involved in induction of apoptosis in $\mathrm{SiHa}$ cells. SiHa cells were treated with live $T$. vaginalis at MOIs of 1,2 , and 5 for $4 \mathrm{~h}$. Live T. vaginalis induced nucleosomal DNA fragmentation at MOI 2 and 5, in a parasiteburden-dependent manner (Figure 2(a)). Cells treated with live $T$. vaginalis also produced cleaved forms of caspase 3 


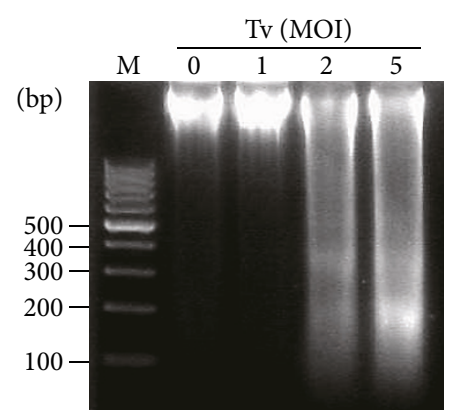

(a)

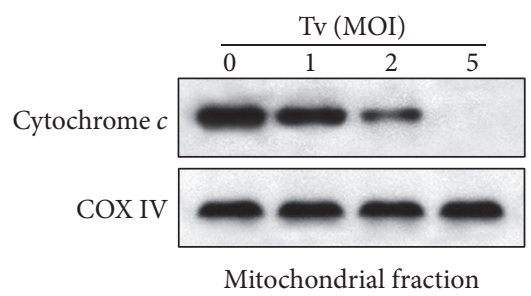

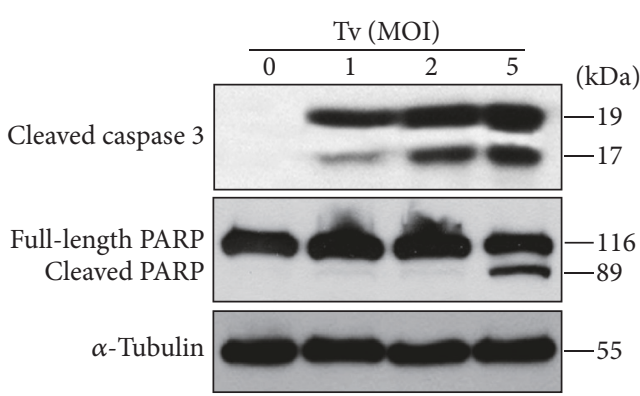

(b)

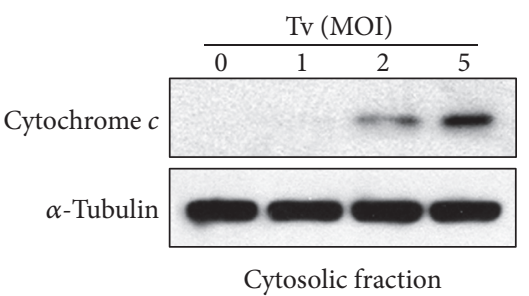

(c)

Figure 2: Mitochondrial apoptosis was induced in SiHa cells following treatment with live T. vaginalis. (a) T. vaginalis induced DNA fragmentation, which is an indicator of apoptosis. (b) T. vaginalis induced the cleavage of caspase 3 and PARP. (c) T. vaginalis induced cytochrome $c$ release from mitochondria into cytosol in parasite-burden-dependent manner. The quality of the fraction experiments was confirmed by assessing the presence of COX IV for the mitochondrial fraction and $\alpha$-tubulin for cytosol fraction. The experiment was repeated three times with similar results. M: marker (100 bp DNA ladder); Tv: Trichomonas vaginalis.

in a parasite-burden-dependent manner and dramatically cleaved PARP at MOI 5 (Figure 2(b)).

Additionally, we examined the release of cytochrome $c$ from the mitochondria as an indicator of involvement of the intrinsic apoptotic pathway. High levels of cytochrome $c$ were present in the mitochondrial fraction of untreated control cells, but not in the cytosolic fraction. In T. vaginalis-treated cells, cytochrome $c$ levels were decreased in mitochondria in a parasite-burden-dependent manner, whereas cytosolic cytochrome $c$ was increased (Figure 2(c)). These results suggest that $T$. vaginalis induces cell death in cervical mucosal epithelium through the intrinsic apoptotic pathway.

\subsection{T. vaginalis Initially Induced Transient Activation of NF- $\kappa B$} by in SiHa Cells, Followed by a Decline in NF- $\kappa$ B Activation. The NF- $\kappa \mathrm{B}$ signaling pathway plays an important role in regulating apoptosis [24]. We used western blotting and luciferase reporter assay to evaluate whether $T$. vaginalis activates the NF- $\kappa$ B signaling pathway in $\mathrm{SiHa}$ cells. In $\mathrm{SiHa}$ cells treated with $T$. vaginalis at MOI 5 , nuclear NF- $\kappa \mathrm{B}$ p65 levels were transiently increased until $1 \mathrm{~h}$ posttreatment. Thereafter, the nuclear NF- $\kappa$ B p 65 level dramatically dropped at $4 \mathrm{~h}$ after treatment. In contrast, cytosol NF- $\kappa \mathrm{B}$ p65 levels decreased until $1 \mathrm{~h}$ after treatment with $T$. vaginalis and then prominently increased at $4 \mathrm{~h}$ after treatment (Figure 3(a)). Further, the NF- $\kappa$ B luciferase reporter assay showed a parasite-dose-dependent reduction in NF- $\kappa$ B activity in T. vaginalis-treated $\mathrm{SiHa}$ cells at $4 \mathrm{~h}$ posttreatment. NF- $\kappa \mathrm{B}$ activity at $T$. vaginalis MOI 5 and MOI 10 was almost totally suppressed (Figure 3(b)). These results indicate that $T$. vaginalis prevents NF- $\kappa \mathrm{B}$ p 65 translocation to the nucleus and suppresses NF- $\kappa \mathrm{B}$ activity in SiHa cells in a parasite-burdendependent manner.

3.4. The ROS Scavenger, NAC, Blocked T. vaginalis-Induced Apoptosis and NF- $\kappa$ B Suppression in SiHa Cells. To block ROS production in $\mathrm{SiHa}$ cells after treatment with T. vaginalis, we used $\mathrm{N}$-acetyl-L-cysteine (NAC) as a ROS scavenger. Before using NAC, we determined its effect on the viability of SiHa cells using an MTT assay. Treatment of SiHa cells with 0.1-1 mM NAC elicited no significant differences in cell viability compared with those in the medium-treated group. $\mathrm{SiHa}$ cells treated with $10 \mathrm{mM}$ NAC showed slightly reduced cell viability (Supplemental Figure 1).

To determine whether ROS are involved in the induction of apoptosis and inactivation of NF- $\kappa \mathrm{B}$ in $T$. vaginalistreated SiHa cells, cells were pretreated with NAC, and then incubated with live $T$. vaginalis. Both intracellular and mitochondrial ROS production were inhibited in $T$. vaginalis-treated $\mathrm{SiHa}$ cells following NAC pretreatment, in a concentration-dependent manner (Figures $4(\mathrm{a})-4(\mathrm{~d})$ ). To examine the relationship between ROS production and apoptosis, DNA fragmentation, caspase activation, cytochrome $c$ release, and Bcl-2 levels were evaluated in T. vaginalis-treated $\mathrm{SiHa}$ cells following pretreatment with NAC. As shown in Figure 5(a), NAC pretreatment strongly suppressed DNA fragmentation induced by $T$. vaginalis and $T$. vaginalis ESP. Similarly, in NAC-pretreated SiHa cells, T. vaginalis-induced cleavage of caspase 3 and PARP were dramatically suppressed (Figure 5(b)). As shown in Figure 5(c), NAC pretreatment significantly increased Bcl-2 protein levels in $T$. vaginalistreated $\mathrm{SiHa}$ cells. In addition, cytochrome $c$ levels in the 


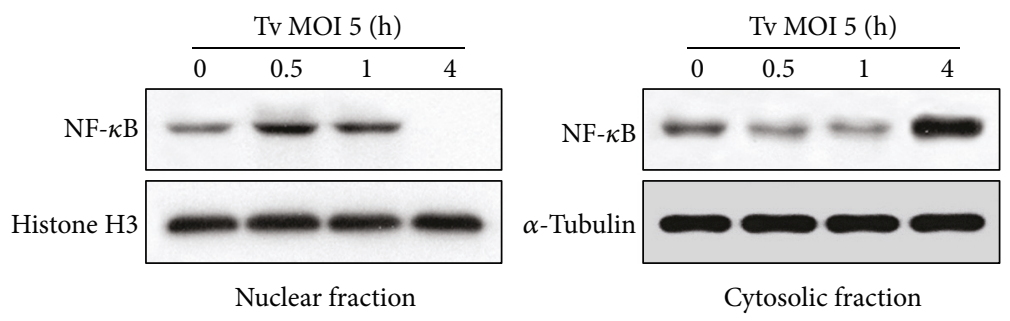

(a)

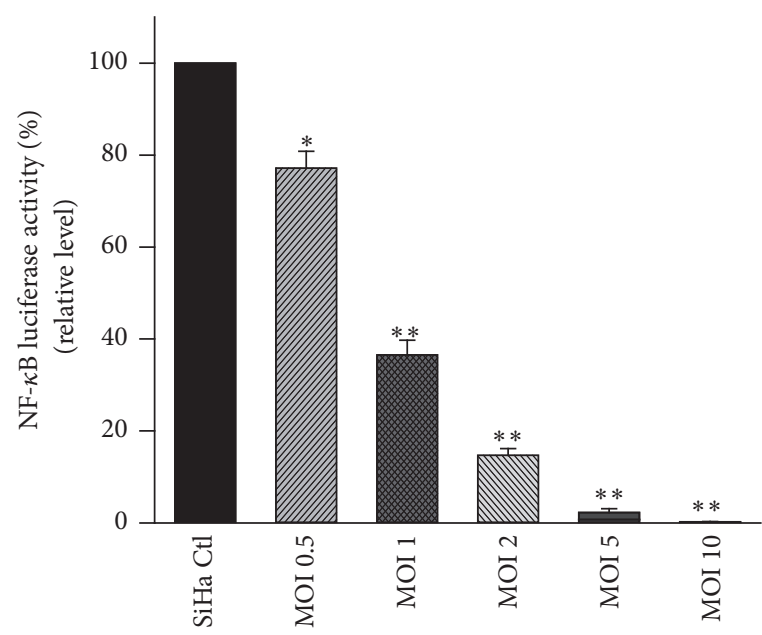

(b)

Figure 3: T. vaginalis initially activated NF- $\kappa$ B in SiHa cells, followed by a decline in NF- $\kappa B$ levels. (a) T. vaginalis transiently induced NF- $\kappa \mathrm{B}$ p65 nuclear translocation in SiHa cells; this was followed by a dramatic decline at $4 \mathrm{~h}$ after treatment. (b) NF- $\kappa \mathrm{B}$ activity of T. vaginalis-treated $\mathrm{SiHa}$ cells were significantly reduced in a parasite-dose-dependent manner at $4 \mathrm{~h}$ posttreatment. The experiment was repeated three times with similar results. ${ }^{*} P<0.05$ versus $\mathrm{SiHa}$ cell control, ${ }^{* *} P<0.01$ versus $\mathrm{SiHa}$ cell control.

mitochondrial fraction was also increased in NAC-pretreated T. vaginalis-treated cells, whereas cytochrome $c$ levels were decreased in the cytosolic fraction (Figure 5(c)). These results suggest that ROS induced by $T$. vaginalis causes oxidative damage to host cell DNA and that ROS act upstream of the signaling molecules such as cytochrome $c$, caspase 3 , and PARP during the induction of apoptosis.

We also determined whether T. vaginalis-induced ROS regulate NF- $\kappa \mathrm{B}$ activity of SiHa cells. Following pretreatment with NAC, reduced nuclear NF- $\kappa$ B p 65 protein levels by $T$. vaginalis were significantly increased in a NACconcentration-dependent manner (Figure 6(a)). In addition, NAC pretreatment was also significantly increased the NF$\kappa \mathrm{B}$ activities in $\mathrm{SiHa}$ cells attenuated by live T. vaginalis and T. vaginalis ESP (Figure 6(b)). These results suggest that ROS production is integral to the NF- $\kappa \mathrm{B}$ signaling pathway; thus T. vaginalis-induced NF- $\kappa \mathrm{B}$ suppression is negated by the suppression of ROS production.

\section{Discussion}

The aim of this study was to determine whether $T$. vaginalisinduced cell apoptosis is mediated by ROS and NF- $\kappa \mathrm{B}$ pathway in SiHa cells. Our results show that T. vaginalis induces intracellular and mitochondrial ROS generation, which causes cytochrome $c$ release into the cytosol, activation of a mitochondria-dependent caspase 3, downregulation of Bcl-2, DNA damage, and, finally, commitment of cells to apoptosis. In addition, $T$. vaginalis initially induced translocation of NF- $\kappa \mathrm{B}$ p65 into nucleus and then blocked these processes at $4 \mathrm{~h}$ after treatment. NF- $\kappa \mathrm{B}$ activity also decreased in a parasite-load-dependent manner at $4 \mathrm{~h}$ after treatment. However, NAC significantly inhibited T. vaginalis-induced nuclear damage, cytochrome $c$ release into the cytoplasm, and activation of cleaved caspase 3 and PARP. Moreover, NAC pretreatment also increased NF- $\kappa$ B p 65 nuclear translocation in a concentration-dependent manner. Thus, our findings indicate that $T$. vaginalis induces $\mathrm{SiHa}$ apoptosis in an ROSdependent manner through NF- $\kappa \mathrm{B}$ suppression.

T. vaginalis, which is known to induce host cell apoptosis in various cell types [5-12], employs various strategies for inducing apoptosis in host cells. Previous reports demonstrated that $T$. vaginalis isolate KT-4 induces apoptosis in human neutrophils by reducing Mcl-1 of Bcl- 2 family proteins [25], and T. vaginalis induces apoptosis in RAW246.7 murine monocyte/macrophage cells via downregulation of $\mathrm{Bcl}-\mathrm{xL}$ [6]. T. vaginalis isolate T016 cleaves Bcl-xL and Mcl-1 in MOI-dependent manner and induces apoptosis in $\mathrm{SiHa}$ cells through the dissociation of $\mathrm{Bcl}-\mathrm{xL} / \mathrm{Bim}$ and $\mathrm{Mcl}-1 / \mathrm{Bim}$ complexes [12]. These reports indicate that Mcl-1, Bcl-xL, and $\mathrm{Bcl}-2$ are all of the members of Bcl-1 family proteins and are well-known inhibitor of apoptosis. During the progress 

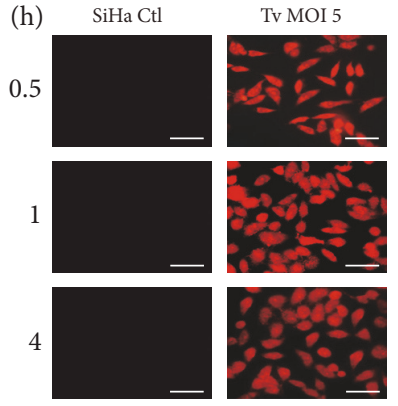
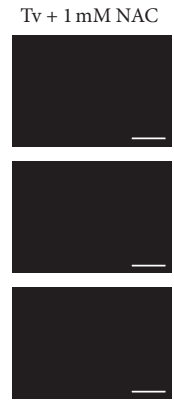

(a)
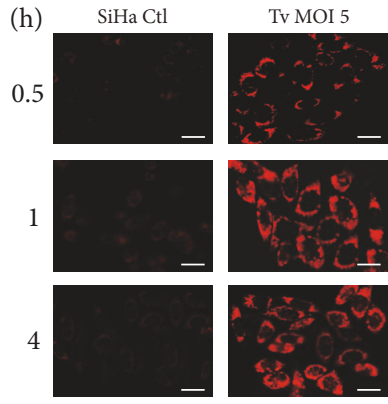

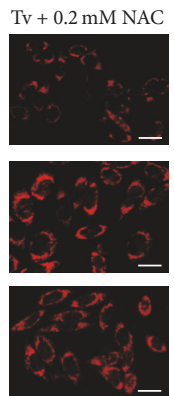

(c)

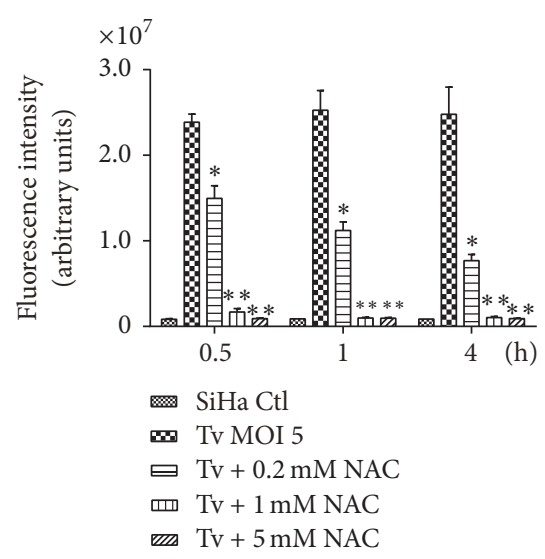

(b)

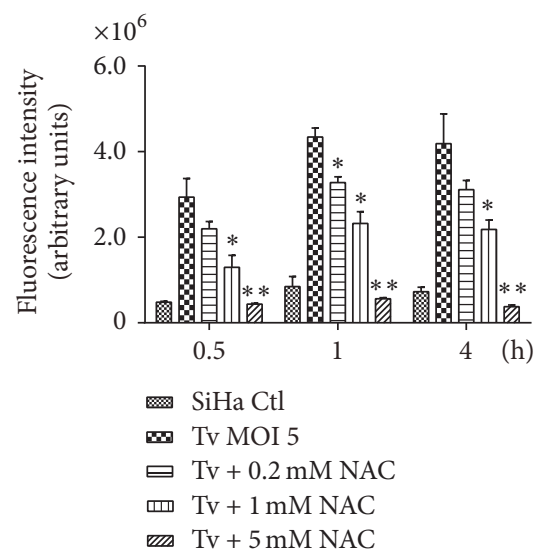

(d)

FIGURE 4: ROS production was blocked in T. vaginalis-treated SiHa cells after pretreatment with N-acetyl-L-cysteine (NAC). (a and b) Intracellular ROS production was detected by dihydroethidium (DHE) fluorescence. Intracellular ROS levels were concentration-dependently inhibited in T. vaginalis-treated SiHa cells following NAC pretreatment. (c and d) Mitochondrial ROS production was detected by MitoSOX reagent. Mitochondrial ROS production was concentration-dependently prevented in T. vaginalis-treated SiHa cells following NAC pretreatment. The experiment was repeated three times with similar results. Scale bars: $100 \mu \mathrm{m} ;{ }^{*} P<0.05$ versus SiHa cell control, ${ }^{* *} P<0.01$ versus $\mathrm{SiHa}$ cell control.

of identifying mitochondrial apoptotic component, each lab was using different targets to excavate noble Bcl-2 family proteins, and that is why there are many reports about different proteins from different cell systems. In the present study, T. vaginalis downregulated Bcl-2 levels, cytochrome $c$ release into the cytosol, and activation of caspase 3 . Thus, our results also clearly implicate the involvement of the mitochondria-dependent intrinsic apoptotic pathway in $T$. vaginalis-induced $\mathrm{SiHa}$ cell death.

It was demonstrated that $T$. vaginalis induces mitochondrial apoptosis through various apoptotic signaling pathways $[6,12,25]$. However, it is poorly understood what trigger molecules are involved in inducing mitochondrial apoptosis of host cells after treatment with $T$. vaginalis. T. vaginalis secretes several hydrolytic enzymes; thus some authors demonstrated that T. vaginalis cysteine proteases induce apoptosis of primary human vaginal epithelial cells [10] and T. vaginalis metalloproteinase induces apoptosis of SiHa cells [12]. Besides the role of T. vaginalis-originated signal in inducing apoptosis of host cells, in addition, ROS and the resulting oxidative stress also play a pivotal role in apoptosis [26]. However, the role of ROS in the apoptosis of human cervical mucosal epithelial cells following stimulation with $T$. vaginalis has not been clarified. In the present study, T. vaginalis increased the intracellular and extracellular ROS levels in SiHa cells, and led to apoptosis. Furthermore, treatment with the ROS scavenger, NAC, prevented T. vaginalismediated apoptosis and modulated the activity of the NF- $\kappa$ B pathway. These results suggest that ROS-dependent caspase 3 activation plays an important role in apoptosis of human cervical mucosal epithelial cells by $T$. vaginalis. Our results are similar to the results of previous studies showing that $T$. vaginalis causes neutrophil apoptosis via NADPH oxidasederived intracellular ROS generation [9].

Next, we were eager to understand the role of ROS in relation to the NK- $\kappa \mathrm{B}$ pathway for apoptosis induction in T. vaginalis-treated $\mathrm{SiHa}$ cells. To resolve this, we adopted two complementary methods: the detection of NF- $\kappa$ B activity by luciferase reporter assay and NF- $\kappa \mathrm{B}$ p65 levels from subcellular components by western blotting. As shown in 


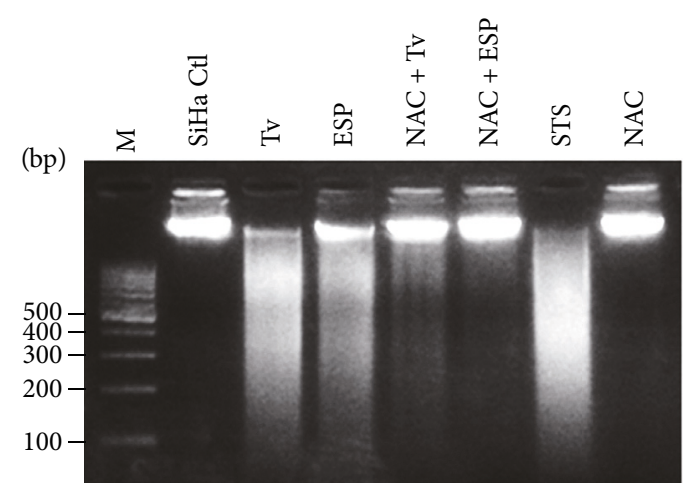

(a)

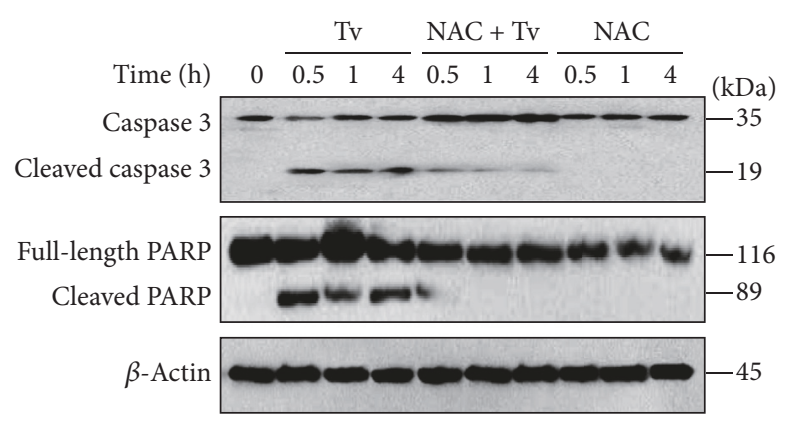

(b)

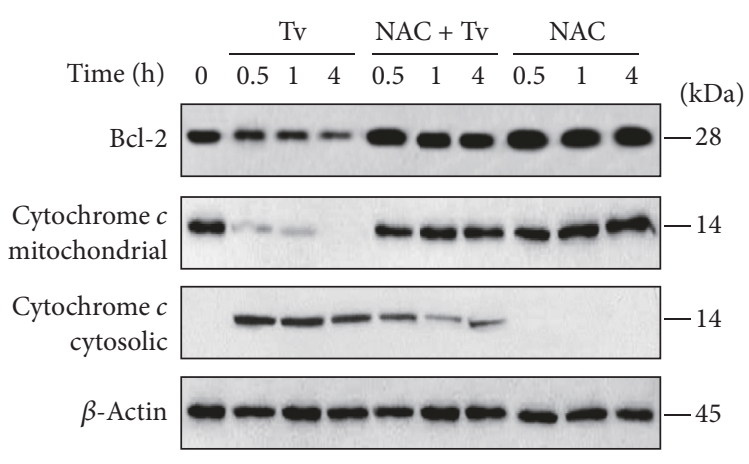

(c)

Figure 5: Apoptosis was prevented in T. vaginalis-treated SiHa cells after pretreatment with NAC. (a) DNA fragmentation was blocked in T. vaginalis-treated SiHa cells by pretreatment with NAC. Lane 1, DNA marker; lane 2, SiHa cell control, lane 3, live T. vaginalis (MOI 5); lane 4, T. vaginalis ESP $(100 \mu \mathrm{g} / \mathrm{mL})$; lane 5 , T. vaginalis (MOI 5) + NAC $(1 \mathrm{mM})$; lane 6, T. vaginalis ESP $(100 \mu \mathrm{g} / \mathrm{mL})+\mathrm{NAC}(1 \mathrm{mM}) ;$ lane 7 , staurosporine (STS, $1 \mu \mathrm{M})$; lane 8, NAC $(1 \mathrm{mM})$. (b) T. vaginalis-induced caspase 3 activation and PARP cleavage in SiHa cells were prevented by NAC pretreatment. (c) T. vaginalis-induced mitochondrial cytochrome $c$ release of SiHa cells was prevented by NAC pretreatment. The experiment was repeated three times with similar results.

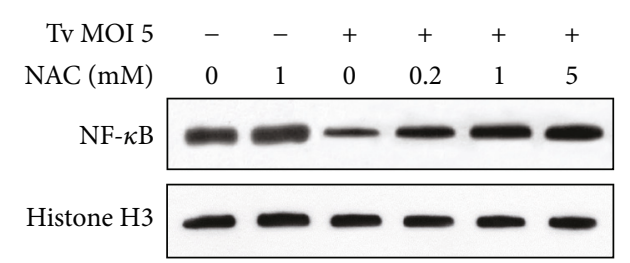

(a)

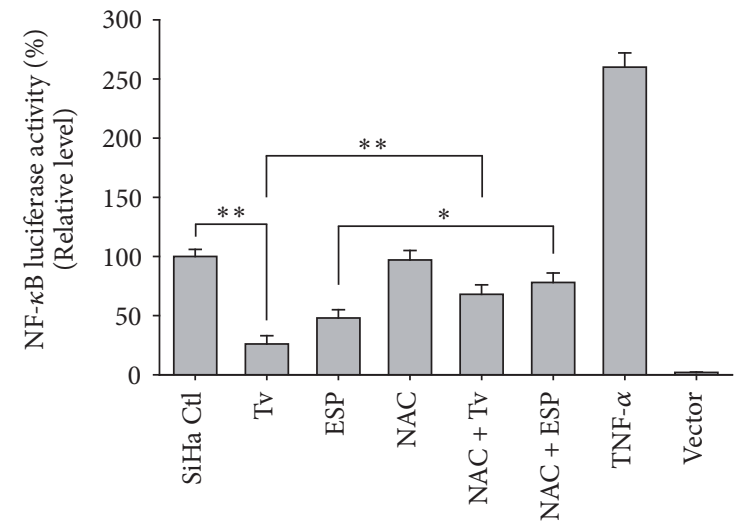

(b)

FiguRE 6: NF- $\kappa$ B activity significantly increased in T. vaginalis-interacted SiHa cells after pretreatment with NAC. (a) Nuclear NF- $\kappa$ B p65 levels were significantly increased in T. vaginalis-treated SiHa cells after NAC pretreatment. (b) NAC pretreatment significantly increased the NF$\kappa \mathrm{B}$ activity attenuated by $T$. vaginalis and T vaginalis ESP. Results are expressed as means \pm standard deviation (SD). The experiment was repeated three times with similar results. ${ }^{* *} P<0.01,{ }^{*} P<0.05$. 


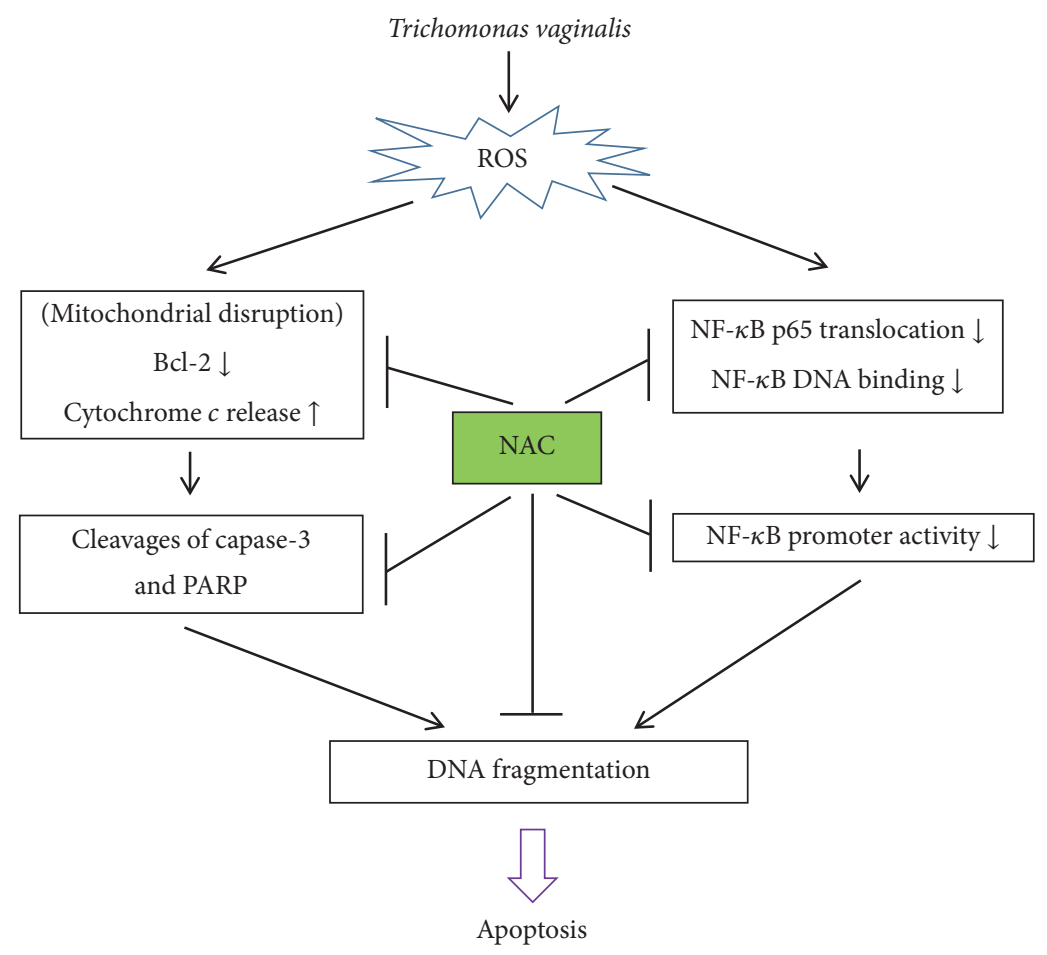

FIGURE 7: Schematic diagram of the T. vaginalis-induced apoptotic pathway in cervical mucosal epithelial SiHa cells. T. vaginalis induces intracellular and mitochondrial ROS production in cervical mucosal epithelial SiHa cells, causing cytochrome c release from the mitochondria and activation of caspase 3. Activation of caspase molecules leads to nuclear fragmentation. T. vaginalis also suppresses NF- $\kappa \mathrm{B}$ nuclear translocation and activity and initiates apoptosis in cervical mucosal epithelial cells.

Figures 3 and 6, T. vaginalis inhibited NF- $\kappa \mathrm{B}$ p65 nuclear translocation and NF- $\kappa \mathrm{B}$ activity in SiHa cells in a parasiteburden-dependent manner. However, NF- $\kappa \mathrm{B}$ inactivation was abrogated by pretreatment with ROS scavenger NAC in T. vaginalis-treated $\mathrm{SiHa}$ cells. Our results suggest that suppression of $\mathrm{NF}-\kappa \mathrm{B}$ activity in T. vaginalis-treated $\mathrm{SiHa}$ cells is implicated in ROS generation. The similar results were shown in the previous studies that host cell apoptosis through inactivation of $\mathrm{NF}-\kappa \mathrm{B}$ activity occurs in human non-small-cell lung cancer cell lines treated with plumbagin [27] and in melanoma A375 cells treated with an extract of C. anthelminticum [28]. Surprising, this study showed that NF- $\kappa \mathrm{B}$ nuclear translocation occurred at $1 \mathrm{~h}$ transiently in T. vaginalis-treated $\mathrm{SiHa}$ cells and then declined at $4 \mathrm{~h}$ posttreatment. Narantsogt et al. [21] also demonstrated that NF- $\kappa \mathrm{B}$ translocation occurs within $1 \mathrm{~h}$ of $T$. vaginalis adhesion and translocation drops dramatically by $8 \mathrm{~h}$ postadhesion. Considering the host-parasite relationship, early activation of NF- $\kappa \mathrm{B}$ nuclear translocation may be related to the protection from apoptotic cell death by $T$. vaginalis; subsequently, $T$. vaginalis inhibits $\mathrm{NF}-\kappa \mathrm{B}$ activity and leads to apoptosis in $\mathrm{SiHa}$ cells as a results of failure of NF- $\kappa \mathrm{B}$ p $50:$ p 65 heterodimers to translocate to the nucleus. However, whether there is a feedback loop between ROS and NF- $\kappa \mathrm{B}$ and the precise regulation mechanism should be further investigated.

In this study, we demonstrated that $T$. vaginalis induces apoptosis in human cervical mucosal epithelial $\mathrm{SiHa}$ cells through an NF- $\kappa \mathrm{B}$-regulated, mitochondria-mediated pathway involving the activation of ROS (Figure 7). The limitation of the current study may be overcome in future investigations by evaluating primary human vaginal epithelial cells (HVECs). Our findings provide novel insights into the pathophysiology of trichomoniasis and mechanisms of action of ROS in the human cervical mucosal epithelium.

\section{Conflicts of Interest}

The authors declare that they have no conflicts of interest.

\section{Authors' Contributions}

Juan-Hua Quan, Byung-Hun Kang, and Jung-Bo Yang contributed equally to this work as co-first authors.

\section{Acknowledgments}

This research was supported by Basic Science Research Program through the National Research Foundation of Korea (NRF) funded by the Ministry of Science, ICT and Future Planning (NRF-2017R1A2B4012822). This work was supported by research fund of Chungnam National University.

\section{Supplementary Materials}

Suppl. 1. Effects of ROS scavenger on the viability of human cervical mucosal epithelial $\mathrm{SiHa}$ cells. $\mathrm{SiHa}$ cells treated with 10 mM ROS scavenger, N-acetyl-L-cysteine (NAC), showed slightly reduced cell viability. Data are shown as the mean \pm 
standard deviation of results for three different experimental wells. (Supplementary Materials)

\section{References}

[1] World Health Organization, Prevalence and Incidence of Selected Sexually Transmitted Infections, Chlamydia Trachomatis, Neisseria gonorrhoeae, Syphilis, and Trichomonas vaginalis: Methods and Results Used by the WHO to Generate 2005 Estimates, World Health Organization, Geneva, Switzerland, 2011.

[2] V. J. Johnston and D. C. Mabey, "Global epidemiology and control of Trichomonas vaginalis," Current Opinion in Infectious Diseases, vol. 21, no. 1, pp. 56-64, 2008.

[3] C. M. Ryan, N. de Miguel, and P. J. Johnson, "Trichomonas vaginalis: current understanding of host-parasite interactions," Essays in Biochemistry, vol. 51, no. 1, pp. 161-175, 2011.

[4] P. C. Guenthner, W. E. Secor, and C. S. Dezzutti, "Trichomonas vaginalis-induced epithelial monolayer disruption and human immunodeficiency virus type 1 (HIV-1) replication: Implications for the sexual transmission of HIV-1," Infection and Immunity, vol. 73, no. 7, pp. 4155-4160, 2005.

[5] Y. S. Ryang, J. H. Chang, and J. Y. Park, "Involvement of MAP Kinases in apoptosis of macrophage treated with Trichomonas vaginalis," Yonsei Medical Journal, vol. 45, no. 4, pp. 751-754, 2004.

[6] J.-H. Chang, Y.-S. Ryang, S.-K. Kim, and J.-Y. Park, "Trichomonas vaginalis-induced apoptosis in RAW264.7 cells is regulated through Bcl- $\mathrm{x}_{\mathrm{L}}$, but not Bcl-2," Parasite Immunology, vol. 26, no. 3, pp. 141-150, 2004.

[7] J.-H. Chang, S.-K. Kim, I.-H. Choi, S.-K. Lee, T. Morio, and E.-J. Chang, "Apoptosis of macrophages induced by Trichomonas vaginalis through the phosphorylation of p38 mitogenactivated protein kinase that locates at downstream of mitochondria-dependent caspase activation," The International Journal of Biochemistry \& Cell Biology, vol. 38, no. 4, pp. 638-647, 2006.

[8] M. H. Ahn, H. O. Song, and J. S. Ryu, “Trichomonas vaginalis-induced neutrophil apoptosis causes anti-inflammatory cytokine production by human monocyte-derived macrophages," Parasite Immunology, vol. 30, no. 8, pp. 410-416, 2008.

[9] H.-O. Song, M.-H. Shin, M.-H. Ahn, D.-Y. Min, Y.-S. Kim, and J.-S. Ryu, "Trichomonas vaginalis: reactive oxygen species mediates caspase-3 dependent apoptosis of human neutrophils," Experimental Parasitology, vol. 118, no. 1, pp. 59-65, 2008.

[10] S. Kummer, G. R. Hayes, R. O. Gilbert, D. H. Beach, J. J. Lucas, and B. N. Singh, "Induction of human host cell apoptosis by Trichomonas vaginalis cysteine proteases is modulated by parasite exposure to iron," Microbial Pathogenesis, vol. 44, no. 3, pp. 197-203, 2008.

[11] U. Sommer, C. E. Costello, G. R. Hayes et al., "Identification of Trichomonas vaginalis cysteine proteases that induce apoptosis in human vaginal epithelial cells," The Journal of Biological Chemistry, vol. 280, no. 25, pp. 23853-23860, 2005.

[12] J. Quan, B. Kang, G. Cha et al., “Trichonomas vaginalis Metalloproteinase Induces Apoptosis of $\mathrm{SiHa}$ Cells through Disrupting the Mcl-1/Bim and Bcl-xL/Bim Complexes," PLoS ONE, vol. 9, no. 10, Article ID el10659, 2014.

[13] C. Gasparini and M. Feldmann, "NF- $\kappa$ B as a target for modulating inflammatory responses," Current Pharmaceutical Design, vol. 18, no. 35, pp. 5735-5745, 2012.
[14] M. Karin and Y. Ben-Neriah, "Phosphorylation meets ubiquitination: the control of NF- $\kappa \mathrm{B}$ activity," Annual Review of Immunology, vol. 18, pp. 621-663, 2000.

[15] J. Li, L. Shen, F. Lu et al., "Plumbagin inhibits cell growth and potentiates apoptosis in human gastric cancer cells in vitro through the NF- $\kappa$ B signaling pathway," Acta Pharmacologica Sinica, vol. 33, no. 2, pp. 242-249, 2012.

[16] J. H. Chang, Y. S. Ryang, T. Morio, S. K. Lee, and E. J. Chang, "Trichomonas vaginalis inhibits proinflammatory cytokine production in macrophages by suppressing NF-kappaB activation," Molecular Microbiology, vol. 18, no. 2, pp. 177-185, 2004.

[17] D. Trachootham, W. Lu, M. A. Ogasawara, N. R.-D. Valle, and P. Huang, "Redox regulation of cell survival," Antioxidants \& Redox Signaling, vol. 10, no. 8, pp. 1343-1374, 2008.

[18] M. L. Circu and T. Y. Aw, "Reactive oxygen species, cellular redox systems, and apoptosis," Free Radical Biology \& Medicine, vol. 48, no. 6, pp. 749-762, 2010.

[19] X. Zhao, X. Tao, L. Xu et al., "Dioscin induces apoptosis in human cervical carcinoma $\mathrm{HeLa}$ and $\mathrm{SiHa}$ cells through ROS-mediated DNA damage and the mitochondrial signaling pathway," Molecules, vol. 21, no. 6, p. E730, 2016.

[20] F. Zeng, L. Tian, F. Liu, J. Cao, M. Quan, and X. Sheng, "Induction of apoptosis by casticin in cervical cancer cells: reactive oxygen species-dependent sustained activation of Jun N-terminal kinase," Acta Biochimica et Biophysica Sinica, vol. 44, no. 5, pp. 442-449, 2012.

[21] G. Narantsogt, A. Min, Y. H. Nam et al., "Activation of MAPK is required for ROS generation and exocytosis in HMC-1 cells induced by Trichomonas vaginalis-derived secretory products," Korean Journal of Parasitology, vol. 53, no. 5, pp. 597-603, 2015.

[22] N. Gu, J. Kim, I. Han et al., “Trichomonas vaginalis induces IL-1 $\beta$ production in a human prostate epithelial cell line by activating the NLRP3 inflammasome via reactive oxygen species and potassium ion efflux," Experimental Parasitology, vol. 76, no. 10, pp. 885-896, 2016.

[23] J. H. Quan, G. H. Cha, W. Zhou, J. Q. Chu, Y. Nishikawa, and Y. H. Lee, "Involvement of PI 3 kinase/Akt-dependent Bad phosphorylation in Toxoplasma gondii-mediated inhibition of host cell apoptosis," Experimental Parasitology, vol. 133, no. 4, pp. 462-471, 2013.

[24] M. Karin, "Nuclear factor- $\kappa \mathrm{B}$ in cancer development and progression," Nature, vol. 441, no. 7092, pp. 431-436, 2006.

[25] J. H. Kang, H. O. Song, J. S. Ryu et al., "Trichomonas vaginalis promotes apoptosis of human neutrophils by activating caspase- 3 and reducing Mcl-1 expression," Parasite Immunology, vol. 28, no. 9, pp. 439-446, 2006.

[26] K. Sinha, J. Das, P. B. Pal, and P. C. Sil, "Oxidative stress: the mitochondria-dependent and mitochondria-independent pathways of apoptosis," Archives of Toxicology, vol. 87, no. 7, pp. 1157-1180, 2013.

[27] T.-P. Xu, H. Shen, L.-X. Liu, and Y.-Q. Shu, "Plumbagin from plumbago zeylanica 1 induces apoptosis in human non-small cell lung cancer cell lines through nf- $\kappa \mathrm{b}$ inactivation," Asian Pacific Journal of Cancer Prevention, vol. 14, no. 4, pp. 2325-2331, 2013.

[28] C. Y. Looi, B. Moharram, M. Paydar et al., "Induction of apoptosis in melanoma A375 cells by a chloroform fraction of Centratherum anthelminticum (L.) seeds involves NF-kappaB, p53 and Bcl-2-controlled mitochondrial signaling pathways," BMC Complementary and Alternative Medicine, vol. 13, no. 1, p. 166, 2013. 

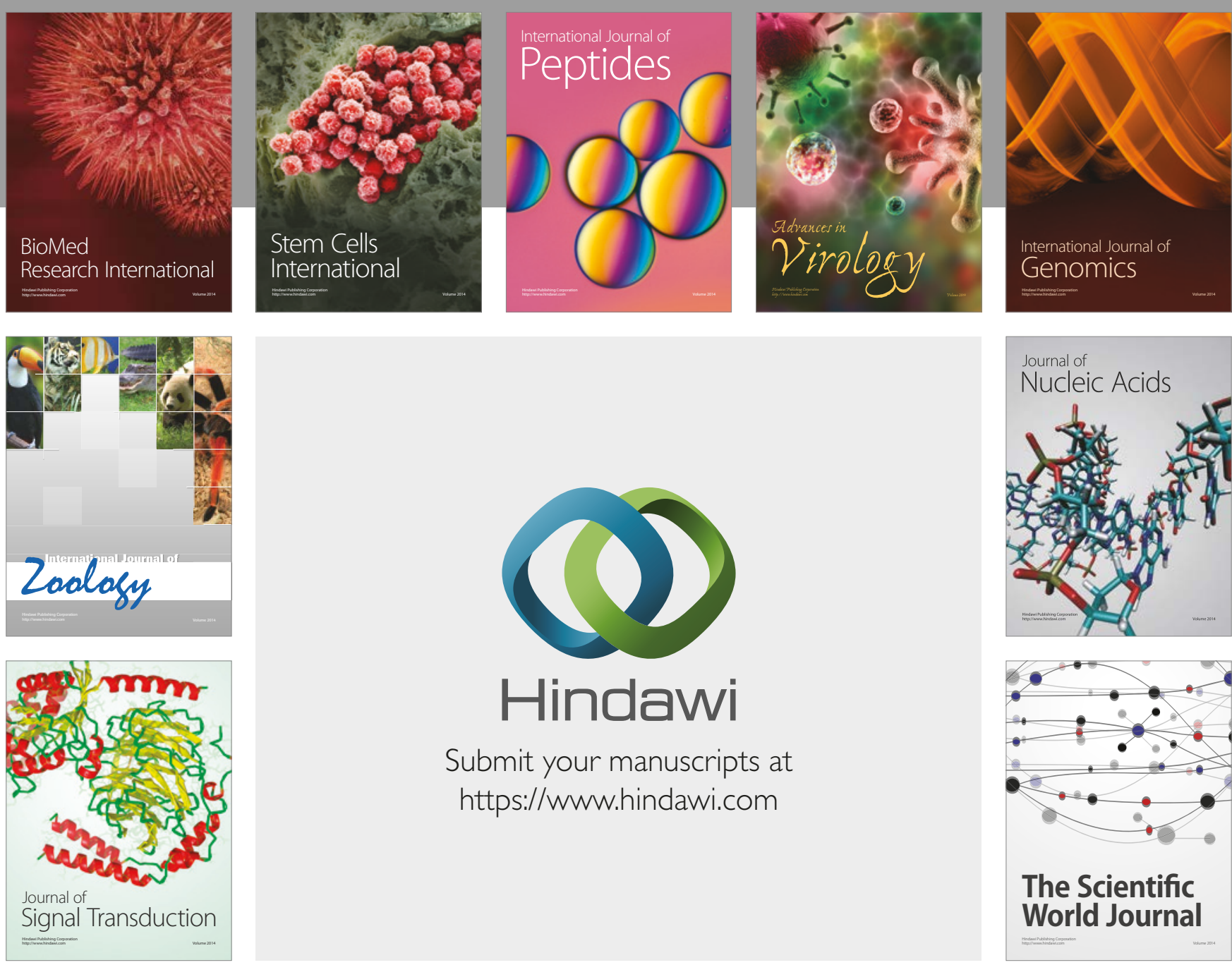

Submit your manuscripts at

https://www.hindawi.com
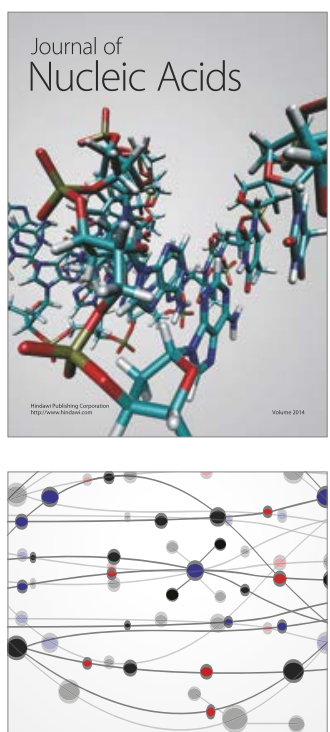

The Scientific World Journal

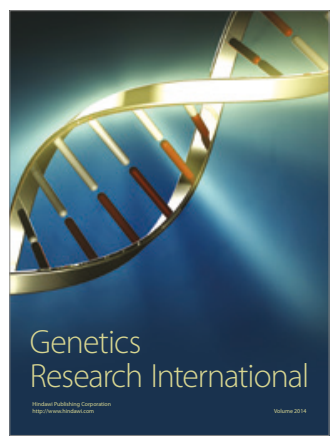

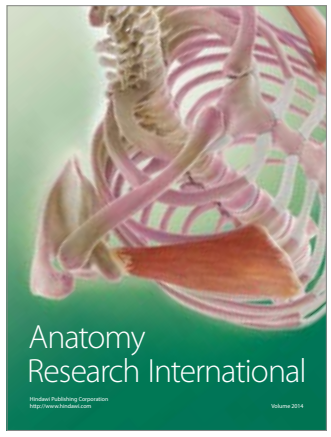

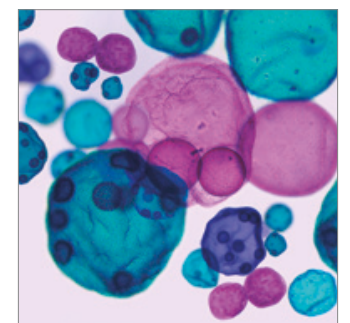

International Journal of Microbiology
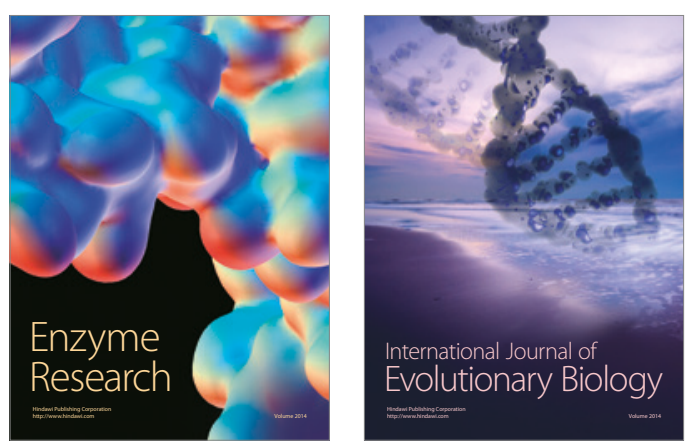
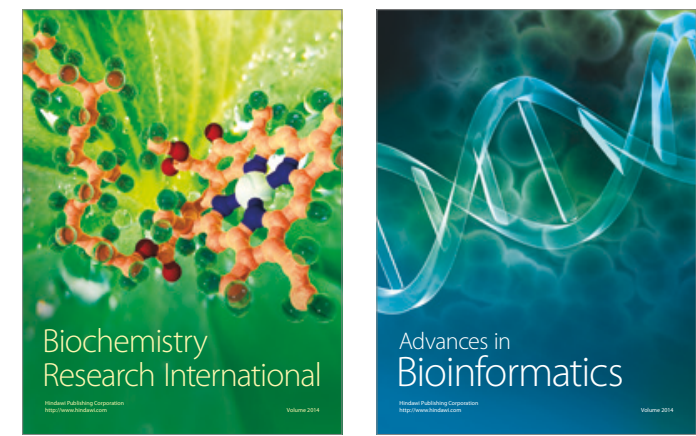

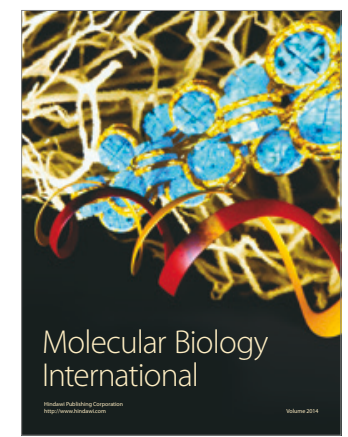

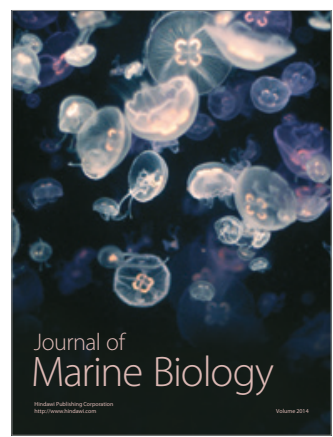

